\title{
LATERAL DISLOCATION OF THE RADIO-HUMERAL JOINT WITH GREENSTICK FRACTURE OF THE UPPER END OF THE ULNA
}

\author{
F. Howard Beddow and P. H. Corkery, Liverpool, England \\ From the Alder Hey Children's Hospital, Liverpool
}

In 1957 Hume described anterior dislocation of the head of the radius associated with undisplaced fracture of the olecranon in children. Of the three cases that he described and illustrated it is our opinion that, whereas Cases 2 and 3 were classical examples of the injury, Case 1 is of an entirely different entity, namely a lateral dislocation of the radio-humeral joint with greenstick fracture of the upper end of the ulna. We have recently encountered two patients with the same injury.
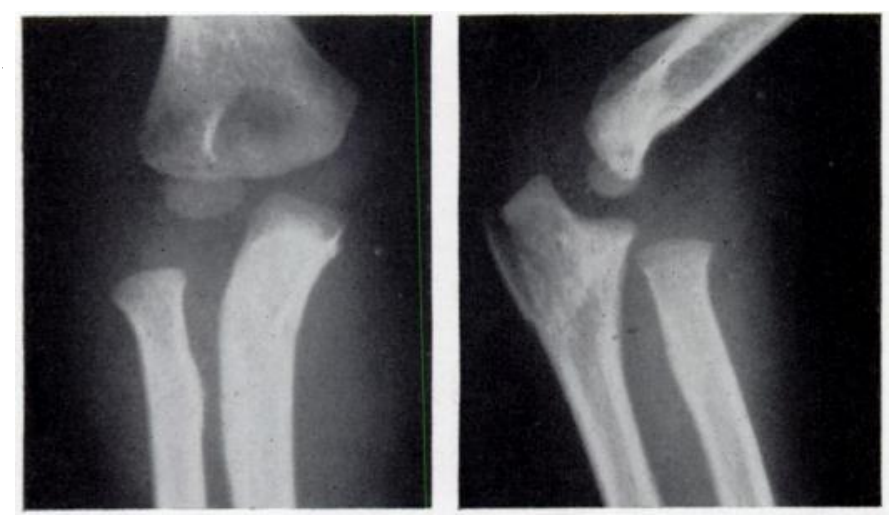

FIG. 1

Case 1-Lateral dislocation of the radio-humeral joint with greenstick fracture of the upper end of the ulna. Before reduction.
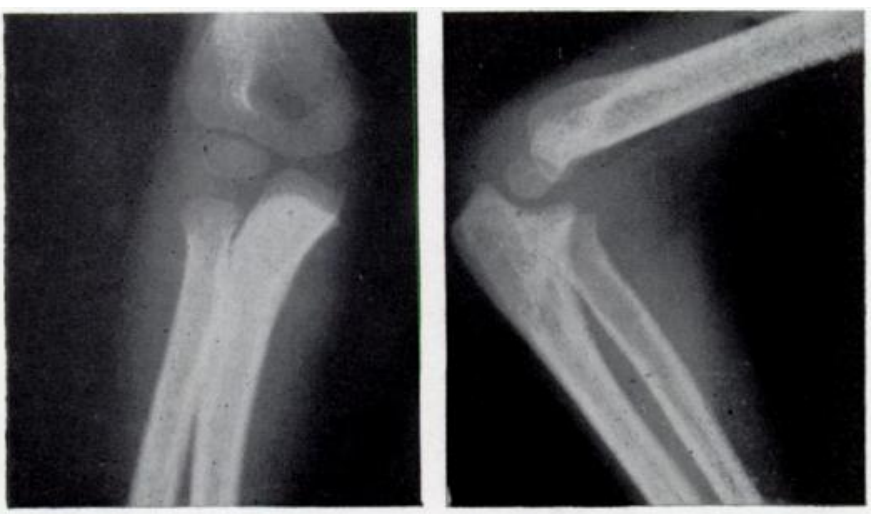

FIG. 2

Case 1-After reduction.
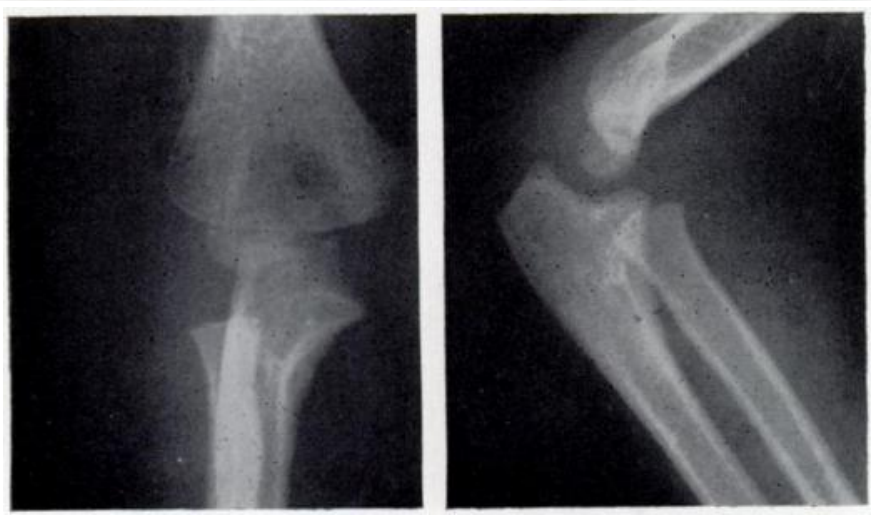

Fig. 3

Case 1-Five months later. 


\section{CASE REPORTS}

Case 1-A boy aged six years sustained an injury to his right elbow in falling from a tricycle. Radiographs showed an oblique greenstick fracture of the upper end of the ulna with lateral dislocation of the radial head (Fig. 1). Reduction was done with ease under general anaesthesia (Fig. 2). The elbow was rested in a collar and cuff sling for four weeks. Normal movement was regained in two further weeks and radiographs at five months were indistinguishable from normal (Fig. 3).

Case 2-A boy aged four years fell from his tricycle and injured his left elbow. Radiographs showed a greenstick fracture of the ulna at the level of the superior radio-ulnar joint, with lateral displacement of the radio-humeral joint (Fig. 4). There was paralysis of the extensor pollicis longus and extensor digitorum communis. Reduction was performed under general anaesthesia (Fig. 5), and the elbow was rested in a collar and cuff sling at 45 degrees for four weeks. Two weeks later there was a full and painless range of elbow movement and a complete

Fig. 4

Case 2-Lateral dislocation of the radio-humeral joint with greenstick fracture of the upper end of the ulna. Before reduction.

FIG. 5

Case 2-After reduction.

FIG. 6

Case 2-Three months later.
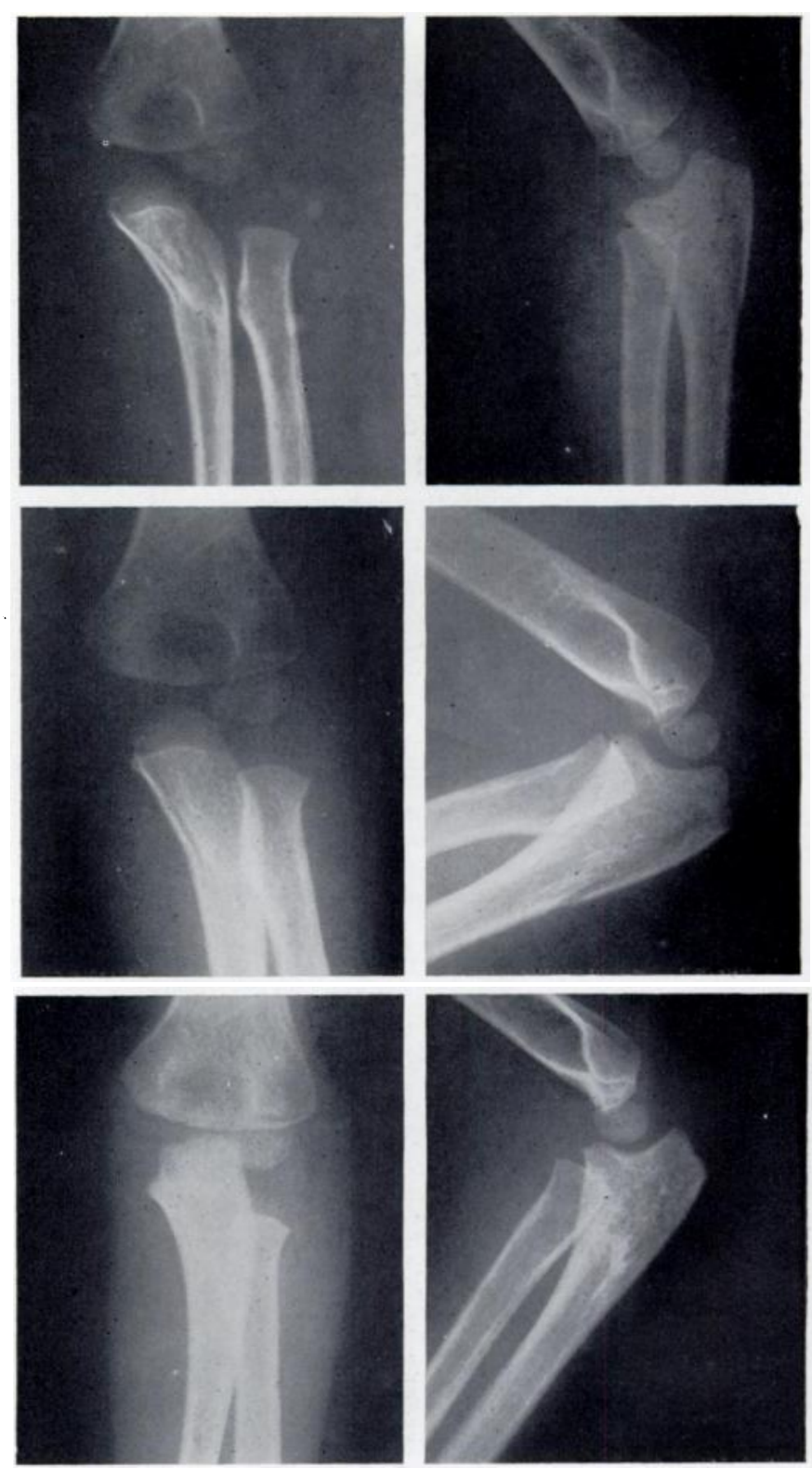
recovery from the radial nerve lesion. Three months later radiographs showed appearances indistinguishable from the normal (Fig. 6).

\section{DISCUSSION}

In our records are radiographs of two further cases. The injury appears to be consistently an oblique greenstick fracture of the upper end of the ulna at the level of the superior radioulnar joint with slight lateral angulation. The lateral view shows the posterior cortex to be split off as a longitudinal "flake." The head of the radius is dislocated laterally at the radio-humeral joint (Figs. 1 and 4).

It is interesting that in Hume's Case 1 and in two of our patients the injury was caused by the child's falling from a cycle.

Reduction was easy under general anaesthesia and consisted simply of correcting the varus deformity by pressure over the head of the radius with the elbow in extension. The elbow was rested at 45 degrees in a collar and cuff sling for four weeks.

One patient had a radial nerve lesion. This was complete at first, but recovery was rapid and complete without any special treatment. It is believed that there was a neurapraxia from traction by the displaced radius on the nerve as it winds through the supinator brevis.

We believe that it is important to draw attention to this lesion, firstly because it may be associated with a radial nerve lesion which (so far as we are aware) has not been described with anterior dislocation, and secondly because immobilisation in plaster-of-Paris appears to be unnecessary.

\section{SUMMARY}

1. Two cases of lateral dislocation of the radio-humeral joint with greenstick fracture of the upper end of the ulna are described.

2. One case was complicated by a radial nerve lesion.

3. Treatment was by reduction under general anaesthesia and resting the elbow in a collar and cuff sling. Full recovery was present in six weeks.

We wish to thank Professor B. L. McFarland for help and advice, and Mr F. C. Dwyer and Mr G. L. Shatwell for permission to publish these cases.

\section{REFERENCE}

Hume, A. C. (1957): Anterior Dislocation of the Head of the Radius Associated with Undisplaced Fracture of the Olecranon in Children. Journal of Bone and Joint Surgery, 39-B, 508. 